\title{
Recent Advances in Management of Anti-Coagulation in Atrial Fibrillation
}

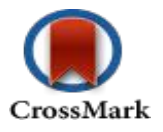

\author{
Prasun Mishra $^{1,4^{*}}$, Shruti Tiwari ${ }^{2}$, Priti Anand ${ }^{3}$, Puneet Anand ${ }^{4 *}$ \\ ${ }^{1}$ Department of Medicine, University Hospitals Case Medical Center, Cleveland, Ohio, USA \\ ${ }^{2}$ Department of Hematology and Oncology, Taussig Cancer Institute- Cleveland Clinic, Cleveland, Ohio, USA \\ ${ }^{3}$ Department of Medicine, Cardiovascular Research Institute, Case Western Reserve University School of Medicine, Cleveland, Ohio, USA \\ ${ }^{4}$ Department of Medicine, Institute for Transformative Molecular Medicine, Case Western Reserve University School of Medicine, Cleveland, \\ Ohio, USA
}

*Corresponding Author: Prasun Mishra, MD, Department of Medicine, University Hospitals Case Medical Center, Cleveland, Ohio 44106, USA. E-mail: pxm260@case.edu

Puneet Anand, PhD, Department of Medicine, Institute for Transformative Molecular Medicine, Case Western Reserve University School of Medicine, Cleveland Ohio 44106, USA. Tel: 216.368.5730; E-mail: pxa100@case.edu

\begin{abstract}
Atrial Fibrillation (AF) is the most common cardiac arrhythmia that is associated with increased irregular atrial contraction, which ultimately leads to rapid ventricular response. Cardioembolic stroke is one of the major complications of AF and it is responsible for significant embolic events in elderly patients. Cardioembolic stroke accounts for up to $20 \%$ of strokes and the incidence increases with advancing age. Current guidelines recommend anticoagulation for AF with high risk factors for emboli based on $\mathrm{CHA}_{2} \mathrm{DS}_{2}$-VASc scoring system. Recently, two major classes of non-vitamin $\mathrm{K}$ oral anti-coagulants (NOACs) have become available for long-term anti-coagulation in AF: Direct Thrombin Inhibitors (DTIs) and Factor Xa Inhibitors. Here we discuss the benefits of NOACs over warfarin in specific patient groups with practical guidance on treatment and monitoring strategies. We will highlight the challenges that clinicians face in the modern era of NOACs. We will further address the strategies for bleeding prevention and treatment and we will conclude by discussing future directions in management of AF.
\end{abstract}

Keywords: Atrial fibrillation; Mechanisms; Management; Therapeutic options

\section{Introduction}

Atrial fibrillation is an arrhythmia characterized by chaotic electrical activity in the atrium arising as a spontaneous ectopy mainly from the pulmonary veins. Other sites of spontaneous ectopy involve superior vena cava, coronary sinus or left atrial appendage. This disorganized electrical activity results in uncoordinated atrial contraction. It is more prevalent in men with increasing age ${ }^{[1]}$. Hypertensive heart disease and coronary heart disease are the most common underlying disorders in patients with AF in developed countries. AF is classified based on $2014 \mathrm{AHA} / \mathrm{ACC} /$ HRS guidelines into paroxysmal AF (AF terminates spontaneously in less than 7 days), persistent AF (AF fails to terminate in 7 days), long standing persistent AF (AF more than 12 months) and permanent AF (AF that is considered not amenable to rhythm control strategy). This classification applies to AF that lasts more than $30 \mathrm{sec}-$
Received date: July 07, 2015

Accepted date: September 28, 2015

Published date: October 05, 2015

Citation: Mishra, P., et al. Recent Advances in Management of Anti-Coagulation in Atrial Fibrillation. (2015) J Heart Cardiol 1(2): 39-43.

DOI: $10.15436 / 2378-6914.15 .013$

onds and is not related to any reversible causes. There is a significant risk of stroke in patients with AF due to formation of clot in left atrial appendage. Cardioembolic stroke is one of the major complications of AF and is responsible for significant embolic events in elderly patients ${ }^{[2]}$. Cardioembolic stroke alone causes up to $20 \%$ of strokes and its incidence increases with age ${ }^{[3]}$. Cardioembolic strokes, which have a high rate of recurrence, exhibit severe presentation and are associated with increased mortality ${ }^{[4,5]}$. Non-valvular $\mathrm{AF}$ is the most common cause of cardioembolic stroke ${ }^{[6,7]}$. AF treatment strategy involves rate control, rhythm control, and anti-coagulation to prevent systemic embolization. Recent advances have been made in oral anti-coagulation strategies 
to prevent cardioembolic stroke in patients with AF. There are several scoring methods developed over the years to estimate the risk of embolic stroke from AF. The latest scoring system is $\mathrm{CHAD}_{2} \mathrm{DS}_{2}-\mathrm{VASc}$ on which current guidelines for anti-coagulation are based. Specifically, this scoring system includes congestive heart failure/left ventricle dysfunction, hypertension, age (more than 65 years), diabetes mellitus, prior stroke/TIA or thromboembolism, peripheral vascular disease or coronary artery disease, and sex; these parameters are considered determinants of thromboembolic stroke ${ }^{[8]}$.

For non-valvular AF with $\mathrm{CHA}_{2} \mathrm{DS}_{2}$-VASc score of 2 or more the benefits of oral anti-coagulation therapy (OAC) exceed risks as described in several studies ${ }^{[9-12]}$. Score of 1 entails various approaches for anti-coagulation depending on several factors including, but not limited to, patient's age, safety, bleeding risks, etc. For patients with score of 0 , the risk of anti-coagulation outweighs the benefits, and therefore the use of anti-coagulation is not recommended. Based on these findings, OAC is class 1 indication for patients with $\mathrm{CHA}_{2} \mathrm{DS}_{2}$-VASc score of 2 or more $^{[13,14]}$.

\section{Benefits of New Non-Vitamin K Oral Anti-Coagulants}

Traditionally, warfarin has been the drug of choice for long term anti-coagulation in AF after initial bridging with heparin. Recently, newer non-vitamin K oral anti-coagulants (NOACs) have become available for long term anti-coagulation in AF. These drugs inactivate both circulating and clot bound activated coagulation factors (Figure). The two major classes of NOAC are direct thrombin inhibitors (DTIs) and factor Xa inhibitors. Parenteral direct thrombin inhibitors include bivalirudin, argatroban and lepirudin, whereas oral direct thrombin inhibitors include dabigatran. Oral factor Xa inhibitors include apixaban, rivaroxaban, and edoxaban. Dabigatran is the only DTI approved for anti-coagulation therapy in $\mathrm{AF}^{[15]}$. Factor $\mathrm{Xa}$ inhibitors approved for anti-coagulation in AF are apixaban, edoxaban and rivaroxaban ${ }^{[16-18]}$, and currently these drugs are approved only for non-valvular AF.

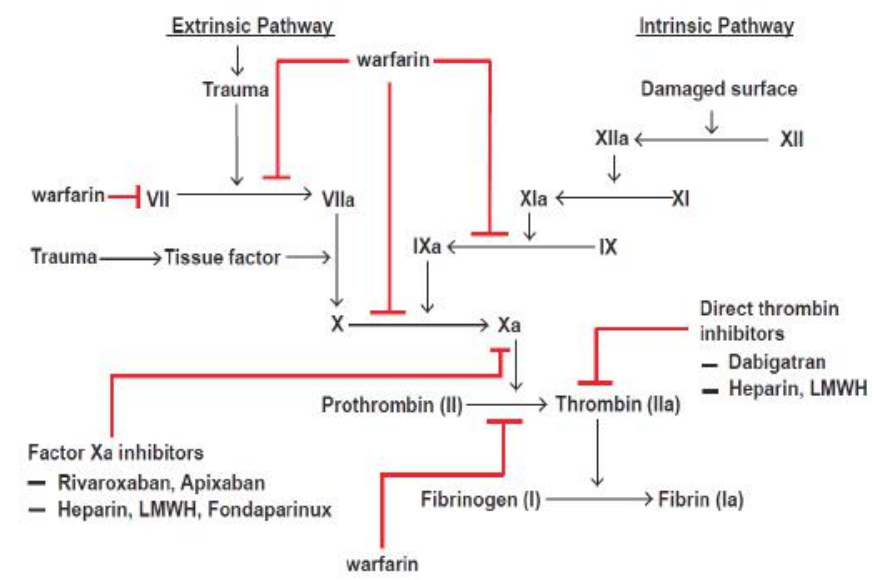

Figure: The coagulation cascade illustrating the tissue injury (extrinsic) and contact (intrinsic) pathways. Factor Xa inhibitors such as Rivaroxaban and apixaban demonstrate selective inhibition of both free and clot-bound factor $\mathrm{Xa}$, whereas other factor $\mathrm{Xa}$ inhibitors such as fondaparinux and heparin/low-molecular-weight heparin (LMWH) indirectly inhibits Xa by activating antithrombin, which is an inhibitor of Xa. Direct thrombin inhibitors such as dabigatran inhibit both free and clot-bound thrombin as well as thrombin-induced platelet aggregation. Warfarin mediates inhibition (indirect) of generation of active forms of factor II, VII, IX, and X.
Accumulating body of evidence indicates a superior risk-benefit profile for NOACs ${ }^{[15-19]}$. Pooled analysis of NOACs trials in the prevention of stroke in patients with AF demonstrated that in comparison to warfarin these agents resulted in significant reductions in stroke or systemic embolic events, reduced intracranial hemorrhage and all-cause mortality (NAOCs and warfarin exhibited similar extent of major bleeding $)^{[20]}$. All NOACs have increased rates of gastrointestinal bleeding with the exception of apixaban. These warfarin alternatives offer several advantages over traditional warfarin treatment. One of the biggest advantages is that unlike warfarin these agents do not need frequent blood tests to adjust dosage, thereby providing a huge convenience to both patients and physicians. Other frequently encountered issue with warfarin is the difficulty in achieving therapeutic INR, which renders the protection offered by anticoagulation ineffective ${ }^{[21,22]}$. These newer drugs mitigate this issue to a large extent as these do not need frequent blood tests and dose adjustments. Also, these drugs do not induce anti-platelet antibodies, thereby making them beneficial in circumstances where heparin is contra-indicated. Consequently, NOACs are preferred for anti-coagulation except in certain circumstances where the patient is already on warfarin with stable INR, valvular AF or when the patient cannot take NOAC due to compliance or cost issues, severe chronic kidney disease, contra-indicated with enzyme inducing anti-epileptic drugs and protease inhibitor anti-retroviral drugs, prosthetic heart valve and pregnancy.

One of the major concerns with use of NOAC is the lack of specific reversal agent. This issue is partly offset by the fact that these drugs have significantly reduced risk of intracranial hemorrhage compared to warfarin and have shorter halve lives, therefore prolonged bleeding is unlikely unless it is due to other underlying conditions or predispositions. In fact, pooled analysis of the trials of the NOACs in the prevention of stroke in patients with AF demonstrated a 52\% reduction in $\mathrm{ICH}$ with these agents versus warfarin (relative risk 0.48; $\mathrm{P}>.0001)^{[20]}$. Evaluation of a US Department of Defense database of a large population of patients (more than 27,000) taking rivaroxaban revealed that major bleeding rate was 2.8 per 100 patient/years $^{[23]}$.

\section{Management of NOACs}

NOACs do not require laboratory monitoring but frequent monitoring of renal function is needed in cases of chronic kidney disease. As far as renal clearance is concerned, DTIs are efficiently cleared by kidneys as compared to factor Xa inhibitors. Specifically, renal clearance rate is $80 \%$ for dabigatran whereas it is $50 \%, 35 \%$ and $27 \%$ for edoxaban, rivaroxaban, and apixaban, respectively. Edoxaban is not recommended in patients with a $\mathrm{CrCl}>95 \mathrm{~mL} / \mathrm{min}$ due to increased risk of ischemic stroke $^{[24]}$. In patients with impaired renal function, the dose of dabigatran, apixaban, and edoxaban is reduced by $50 \%$ whereas the dose of rivaroxaban is reduced by $25 \%$. Rivaroxaban and edoxaban are dosed once daily, whereas dabigatran and apixaban are dosed twice daily. Besides creatinine clearance, apixaban dose reduction is also based on patient's weight and age. Of note, there are differences in the uptake of the NOACs based on whether or not the patient takes them with food. This is especially important in the case of rivaroxaban because it has been shown that this drug is absorbed better when taken with food ${ }^{[25]}$. There are no specific recommendations for apixaban or edoxaban; however, for dabigatran the risk of side effects (particular- 
ly dyspepsia) is reduced if the drug is taken with food.

NOACs have demonstrated a significantly faster onset of therapeutic effect with significantly shorter half-life compared to warfarin. Although NOACs appear to represent alternatives with more straight forward management than that of warfarin, there are many important issues that need to be addressed in the use of these drugs. One of the major issues is management of bleeding. It is very difficult to assess risk of significant bleeding as the dose response assessment is a big challenge with NOACs due to lack of commercially available assays. Efforts are under way to discover novel agents (to be used as antidotes) or reversal agents (to correct the anticoagulant effects of NOACs) that are safe and effective for routine use. Currently, management of bleeding episodes in patients taking NOACs include stopping the drug and providing supportive care. In life threatening cases, activated and inactivated prothrombin complex concentrates such as FEIBA (factor VIII inhibitor bypassing agent) or K centra can be given ${ }^{[26}$. Anti-fibrinolytic agent (e.g. tranexamic acid) may also be used in less severe cases. Andexanet Alfa, which is a recombinant modified Factor Xa molecule, is currently being developed as an antidote for patients receiving a Factor $\mathrm{Xa}$ inhibitor who suffer a major bleeding episode. A Phase 3 study, the ANNEXA-A (Andexanet Alfa a Novel Antidote to the Anticoagulant Effects of FXA Inhibitors-Apixaban), was presented at the American Heart Association 2014 Scientific Session and it showed a rapid normalization of anti-factor Xa and thrombin levels [ANNEXA ${ }^{\text {TM }}$ A: a Phase 3 randomized, double-blind, placebo-controlled trial, demonstrating reversal of apixaban-induced anticoagulation in older subjects by Andexanet Alfa (PRT064445), a universal antidote for factor Xa (fXa) inhibitors (abstract-Circulation 2014;130: 2105-26]. This was followed by a phase 3 trial ANNEXA-R demonstrating safety and efficacy of Andexanet Alfa and showing a rapid near complete reversal of anticoagulation effects of rivaroxaban. A specific antidote for dabigatran is Idarucizumab, which has been developed by Boehringer Ingelheim. Another Phase 3 study, the RE-VERSE AD (A Study of the Reversal Effects of Idarucizumab on Active Dabigatran), is already under way in patients with dabigatran-induced bleeding or those requiring emergency surgery.

Another issue in the use of NOACs is peri-procedural management of these drugs. Expert consensus and guidelines recommend administration of anticoagulation at least during and after procedures ${ }^{[27,28]}$. Studies in patients with AF undergoing ablation have shown that major bleeding and stroke/thromboembolic rates were similar with apixaban, rivaroxaban, and warfarin $^{[29,30]}$. Several post-hoc analyses of phase 3 trials suggest equivalence between warfarin and the newer drugs in minority of patients who underwent cardioversion and/or $\mathrm{RFA}^{[31-33]}$. Multiple observational studies have shown that newer drugs are equivalent to warfarin ${ }^{[34-37]}$. Recently published VENTURE-AF trial is the first randomized controlled trial of a non-vitamin $\mathrm{K}$ oral anticoagulant versus warfarin in patients with AF undergoing ablation ${ }^{[28]}$. Patients enrolled in the VENTURE-AF trial were randomized to receive uninterrupted warfarin (target international normalized ratio $=2-3$ ) versus uninterrupted rivaroxaban (dosage $20 \mathrm{mg}$ ) ${ }^{[29]}$. The results provide conclusive evidence of safety of using rivaroxaban in patients with AF undergoing catheter ablation. This study demonstrated that the use of uninterrupted oral rivaroxaban was feasible and event rates were similar to those obtained with uninterrupted warfarin therapy in non valvular AF undergoing catheter ablation.

\section{Future Directions for Stroke Prevention in AF Patients}

There are several other alternative anticoagulants (factor Xa inhibitors, betrixaban and darexaban, and the direct thrombin inhibitor, AZD0837) which are in the development stage that aim to offer better safety profile and efficacy. A variety of anticoagulant strategies (polyphosphate inhibitors, factor VIII inhibitor, thrombomodulin, tissue factor pathway inhibitors, factor IXa inhibitor, factor XI inhibitor, factor XIIa inhibitor) targeting the above mentioned pathways (and other pathways not described here) in the coagulation cascade are in developmental stages. Further research is needed to determine the exact pathways involved in embolic stroke in AF patients. One of the major contributions to the pathogenesis in causing cardioembolic stroke is the stagnation of blood in left atrial appendage (LAA) and resultant formation of blood clot in $\mathrm{LAA}^{[38]}$. Hence, closure of LAA and isolating it from the main left atrial chamber may prevent formation of blood clots and cardioembolic strokes. Several surgical and non-surgical modalities are underway to prevent formation of blood clot in LAA. One of this modality involves placing a closure device in LAA and preventing blood clot formation. A similar device (Watchman device) was shown to be non-inferior to warfarin therapy in prevention of cardioembolic strokes ${ }^{[39]}$. Several other modalities to control blood clot formation and stroke in AF patients involve development of new drugs and procedures that target AF itself or to control rate and rhythm. Recently, major advances have been made in non-pharmacologic control of AF using techniques involving radio-frequency (RF) ablation or non-RF ablation of AF. In fact, catheter ablation has been shown to be more efficacious than anti-arrhythmic drug in maintaining normal sinus rhythm ${ }^{[40]}$.

\section{Conclusion}

The development of NOACs is a major breakthrough in anti-coagulation treatment for stroke prevention in AF. These newer anti-coagulants have greater efficacy with a more reliable and predictable anticoagulant effect without the need for laboratory monitoring, thereby offering a more convenient option for patients and physicians. However, these medications are expensive and have several unique characteristics, and therefore require utmost caution from physicians regarding prescription information based on latest guidelines and product packet inserts.

\section{References}

1. Go, A.S., Hylek, E.M., Phillips, K.A., et al. Prevalence of diagnosed atrial fibrillation in adults: national implications for rhythm management and stroke prevention: the Anticoagulation and Risk Factors in Atrial Fibrillation (ATRIA) Study. (2001) JAMA 285(18): 2370-2375.

2. Albers, G.W., Dalen, J.E., Laupacis, A., et al. Antithrombotic therapy in atrial fibrillation. (2001) Chest 119(1 Suppl): 194S-206S.

3. Palacio, S., Hart, R.G. Neurologic manifestations of cardiogenic embolism: an update. (2002) Neurol Clin 20(1): 179-193.

4. Eriksson, S.E., Olsson, J.E. Survival and recurrent strokes in patients with different subtypes of stroke: a fourteen-year follow-up study. (2001) Cerebrovasc Dis 12(3): 171-180.

5. Kolominsky-Rabas, P.L., Weber, M., Gefeller, O., et al. Epidemiology of ischemic stroke subtypes according to TOAST criteria: incidence, 
recurrence, and long-term survival in ischemic stroke subtypes: a population-based study. (2001) Stroke 32(12): 2735-2740.

6. Bogousslavsky, J., Cachin, C., Regli, F., et al. Cardiac sources of embolism and cerebral infarction--clinical consequences and vascular concomitants: the Lausanne Stroke Registry. (1991) Neurology 41(6): 855-859.

7. Caplan, L.R. Brain embolism, revisited. (1993) Neurology 43(7): 1281-1287.

8. Lip, G.Y., Halperin, J.L. Improving stroke risk stratification in atrial fibrillation. (2010) Am J Med 123(6): 484-488.

9. Friberg, L., Rosenqvist, M., Lip, G.Y. Net clinical benefit of warfarin in patients with atrial fibrillation: a report from the Swedish atrial fibrillation cohort study. (2012) Circulation 125(19): 2298-2307.

10. Singer, D.E., et al. The net clinical benefit of warfarin anticoagulation in atrial fibrillation. (2009) Ann Intern Med 151(5): 297-305.

11. Olesen, J.B., Lip, G.Y., Lindhardsen, J., et al. Risks of thromboembolism and bleeding with thromboprophylaxis in patients with atrial fibrillation: A net clinical benefit analysis using a 'real world' nationwide cohort study. (2011) Thromb Haemost 106(4): 739-749.

12. Banerjee, A., Lane, D.A., Torp-Pedersen, C., et al., Net clinical benefit of new oral anticoagulants (dabigatran, rivaroxaban, apixaban) versus no treatment in a 'real world' atrial fibrillation population: a modelling analysis based on a nationwide cohort study. (2012) Thromb Haemost 107(3): 584-589.

13. Anderson, J.L., Halperin, J.L., Albert, N.M., et al. Management of patients with atrial fibrillation (compilation of $2006 \mathrm{ACCF} / \mathrm{AHA} / \mathrm{ESC}$ and 2011 ACCF/AHA/HRS recommendations): a report of the American College of Cardiology/American Heart Association Task Force on Practice Guidelines. (2013) J Am Coll Cardiol 61(18): 1935-1944.

14. Camm, A.J., et al. 2012 focused update of the ESC Guidelines for the management of atrial fibrillation: an update of the 2010 ESC Guidelines for the management of atrial fibrillation. Developed with the special contribution of the European Heart Rhythm Association. (2012) Eur Heart J 33(21): 2719-2747.

15. Connolly, S.J., Lip, G.Y., De Caterina, R., et al. Dabigatran versus warfarin in patients with atrial fibrillation. (2009) N Engl J Med 361(12): 1139-1151.

16. Granger, C.B., Alexander, J.H., McMurray, J.J., et al. Apixaban versus warfarin in patients with atrial fibrillation. (2011) N Engl J Med 365(11): 981-992.

17. Patel, M.R., Mahaffey, K.W., Garg, J., et al. Rivaroxaban versus warfarin in nonvalvular atrial fibrillation. (2011) N Engl J Med 365(10): 883-891.

18. Giugliano, R.P., Ruff, C.T. Braunwald, E., et al. Edoxaban versus warfarin in patients with atrial fibrillation. (2013) N Engl J Med 369(22): 2093-104.

19. Torinuki, W., Tagami, H. The role of complement in UVB-induced inflammation. (1986) Acta Derm Venereol 66(5): 386-390.

20. Ruff, C.T., Giugliano, R.P., Braunwald, E., et al. Comparison of the efficacy and safety of new oral anticoagulants with warfarin in patients with atrial fibrillation: a meta-analysis of randomised trials. (2014) Lancet 383(9921): 955-962.

21. Connolly, S.J., Pogue, J., Eikelboom, J., et al. Benefit of oral anticoagulant over antiplatelet therapy in atrial fibrillation depends on the quality of international normalized ratio control achieved by centers and countries as measured by time in therapeutic range. (2008) Circulation 118(20): 2029-2037.

22. Morgan, C.L., McEwan, P., Tukiendorf, A., et al. Warfarin treatment in patients with atrial fibrillation: observing outcomes associated with varying levels of INR control. (2009) Thromb Res 124(1): 37-41.

23. Tamayo, S., Frank Peacock, W., Patel, M. et al. Characterizing major bleeding in patients with nonvalvular atrial fibrillation: a pharmacovigilance study of 27467 patients taking rivaroxaban. (2015) Clin Cardiol 38(2): 63-68.

24. Stampfuss, J., Kubitza D Becka, M., et al. The effect of food on the absorption and pharmacokinetics of rivaroxaban. (2013) Int J Clin Pharmacol Ther 51(7): 549-561.
25. Marlu, R., Hodaj, E., Paris, A., et al. Effect of non-specific reversal agents on anticoagulant activity of dabigatran and rivaroxaban: a randomised crossover ex vivo study in healthy volunteers. (2012) Thromb Haemost 108(2): 217-24.

26. Fuster, V., Rydén, L.E., Cannom, D.S. et al. 2011 ACCF/AHA/HRS focused updates incorporated into the ACC/AHA/ESC 2006 guidelines for the management of patients with atrial fibrillation: a report of the American College of Cardiology Foundation/American Heart Association Task Force on practice guidelines. (2011) Circulation 123(10): e269-367.

27. Calkins, H., Kuck, K.H., Cappato, R., et al. 2012 HRS/EHRA/ ECAS expert consensus statement on catheter and surgical ablation of atrial fibrillation: recommendations for patient selection, procedural techniques, patient management and follow-up, definitions, endpoints, and research trial design: a report of the Heart Rhythm Society (HRS) Task Force on Catheter and Surgical Ablation of Atrial Fibrillation. Developed in partnership with the European Heart Rhythm Association (EHRA), a registered branch of the European Society of Cardiology (ESC) and the European Cardiac Arrhythmia Society (ECAS); and in collaboration with the American College of Cardiology (ACC), American Heart Association (AHA), the Asia Pacific Heart Rhythm Society (APHRS), and the Society of Thoracic Surgeons (STS). Endorsed by the governing bodies of the American College of Cardiology Foundation, the American Heart Association, the European Cardiac Arrhythmia Society, the European Heart Rhythm Association, the Society of Thoracic Surgeons, the Asia Pacific Heart Rhythm Society, and the Heart Rhythm Society. (2012) Heart Rhythm 9(4): 632-696 e21.

28. Cappato, R., Marchlinski, F.E., Hohnloser, S.H., et al. Uninterrupted rivaroxaban vs. uninterrupted vitamin $\mathrm{K}$ antagonists for catheter ablation in non-valvular atrial fibrillation. (2015) Eur Heart J 36(28): 1805-1811.

29. Bin Abdulhak, A.A., Khan, A.R., Tleyjeh, I.M., et al. Safety and efficacy of interrupted dabigatran for peri-procedural anticoagulation in catheter ablation of atrial fibrillation: a systematic review and meta-analysis. (2013) Europace 15(10): 1412-1420.

30. Nagarakanti, R., Ezekowitz, M.D., Oldgren, J., et al. Dabigatran versus warfarin in patients with atrial fibrillation: an analysis of patients undergoing cardioversion. (2011) Circulation 123(2): 131-136.

31. Flaker, G., Lopes, R.D., Al-Khatib, S.M., et al. Efficacy and safety of apixaban in patients after cardioversion for atrial fibrillation: insights from the ARISTOTLE Trial (Apixaban for Reduction in Stroke and Other Thromboembolic Events in Atrial Fibrillation). (2014) J Am Coll Cardiol 63(11): 1082-1087.

32. Piccini, J.P., Stevens, S.R., Lokhnygina, Y., et al. Outcomes after cardioversion and atrial fibrillation ablation in patients treated with rivaroxaban and warfarin in the ROCKET AF trial. (2013) J Am Coll Cardiol 61(19): 1998-2006.

33. Providencia, R., Albenque, J.P., Combes, S., et al. Safety and efficacy of dabigatran versus warfarin in patients undergoing catheter ablation of atrial fibrillation: a systematic review and meta-analysis. (2014) Heart 100(4): 324-335.

34. Steinberg, B.A., Hasselblad, V., Atwater, B.D. et al. Dabigatran for periprocedural anticoagulation following radiofrequency ablation for atrial fibrillation: a meta-analysis of observational studies. (2013) J Interv Card Electrophysiol 37(3): 213-221.

35. Lakkireddy, D., Reddy, Y.M., Di Biase, L., et al. Feasibility and safety of uninterrupted rivaroxaban for periprocedural anticoagulation in patients undergoing radiofrequency ablation for atrial fibrillation: results from a multicenter prospective registry. (2014) J Am Coll Cardiol 63(10): 982-988.

36. Lakkireddy, D., Reddy, Y.M., Di Biase, L., et al. Feasibility and safety of dabigatran versus warfarin for periprocedural anticoagulation in patients undergoing radiofrequency ablation for atrial fibrillation: results from a multicenter prospective registry. (2012) J Am Coll Cardiol 59(13): 1168-1174.

37. Transesophageal echocardiographic correlates of thromboembolism in high-risk patients with nonvalvular atrial fibrillation. The Stroke Pre- 
vention in Atrial Fibrillation Investigators Committee on Echocardiography. (1998) Ann Intern Med 128(8): 639-647.

38. Holmes, D.R., Reddy, V.Y., Turi, Z.G., et al. Percutaneous closure of the left atrial appendage versus warfarin therapy for prevention of stroke in patients with atrial fibrillation: a randomised non-inferiority trial. (2009) Lancet 374(9689): 534-542.

39. Lee, R., Kruse. J., McCarthy, P.M. Surgery for atrial fibrillation. (2009) Nat Rev Cardiol 6(8): 505-513.

40. Savaysa (edoxaban) [prescribing information]. (2015) Parsippany, NJ: Daiichi Sankyo.

Journal ISSN: 2378-6914 (online)

Journal Title: Journal of Heart and Cardiology Short title : J Heart Cardiol
Ommega Online Publishers

Journal E-mail: cardiology@ommegaonline.com Website: www.ommegaonline.org 